BNL-

$\mathrm{UC}-414$

AGS/AD/95-1

FORMAL

NUMERICAL STUDIES OF SIBERIAN SNAKES and SPIN ROTATORS FOR RHIC

\author{
Alfredo Luccio
}

April 17, 1995

\begin{abstract}
ALTERNATING GRADIENT SYNCHROTRON DEPARTMENT
BROOKHAVEN NATIONAL LABORATORY ASSOCIATED UNIVERSITIES, INC. UPTON, LONG ISLAND, NEW YORK
\end{abstract}

UNDER CONTRACT NO. DE-AC02-76CH00016 WITH THE UNITED STATES DEPARTMENT OF ENERGY 


\section{DISCLAIMER}

This report was prepared as an account of work sponsored by the United States Government. Neither the United States nor the United States Department of Energy, nor any of their employees, nor any of their contractors, subcontractors, or their employees, makes any warranty, express or implied, or assumes any legal liability or responsibility for the accuracy, completeness, or usefulness of any information, apparatus, product or process disclosed, or represents that its use would not infringe privately owned rights. 


\section{DISCLAIMER}

Portions of this document may be illegible in electronic image products. Images are produced from the best available original document. 


\title{
NUMERICAL STUDIES OF SIBERIAN SNAKES AND SPIN ROTATORS FOR RHIC
}

\author{
Alfredo Luccio
}

\section{Introduction}

For the program of polarized protons in RHIC, two Siberian snakes and four spin rotators per ring will be used [Roser, 1]. The Snakes will produce a complete spin flip. Spin Rotators, in pairs, will rotate the spin from the vertical direction to the horizontal plane at a given insertion, and back to the vertical after the insertion. Snakes, $180^{\circ}$ apart and with their axis of spin precession at $90^{\circ}$ to each other, are an effective means to avoid depolarization of the proton beam in traversing resonances. Rotators are needed to study proton collisions with the direction of the spin in the horizontal plane.

Classical snakes and rotators are made with magnetic solenoids or with a sequence of magnetic dipoles with fields alternately directed in the radial and vertical direction [2]. Another possibility is to use helical magnets, essentially twisted dipoles, in which the field, transverse to the axis of the magnet, continuously rotates as we proceed along it. After some comparative studies, we decided to adopt for RHIC an elegant solution with four helical magnets both for the snakes and the rotators proposed by Shatunov and Ptitsin [3]. In order to simplify the construction of the magnets and to minimize their cost we will make use of four identical super conducting helical modules for each device. Snakes will be built with four right-handed helices. Spin rotators with two right-handed and two left-handed helices. The maximum field will be limited to 4 Tesla.

While small bore helical undulators have been built for free electron lasers, large super conducting helical magnets have not been built yet. In spite of this difficulty, our choice is dictated by some distinctive advantages of helical over more conventional transverse snakes/rotators:

(i) the devices are modular, i.e. they can be built with arrangements of identical modules,

(ii) the maximum orbit excursion in the magnet is smaller,

(iii) orbit excursion is independent from the separation between adjacent magnets,

(iv) they allow an easier control of the spin rotation and the orientation of the spin precession axis.

Since we want to use the same standard cryostats of the RHIC dipoles, the length of the snakes/rotators should be limited to 12 meters. Accordingly, we have chosen $2.4 \mathrm{~m}$ as the length of each module, nominally separated by a distance of $32 \mathrm{~cm}$. The magnet bore as been chosen as $10 \mathrm{~cm}$. The magnet separation is not critical, because of the property (iii) above. The bore is adequate for the resulting orbit deformation, as it will be shown.

A first calculation of the properties of a four helix spin rotator can be made with the use of appropriate matrices that describe the spin rotation through the four modules. In a similar way the beam optical properties of the device can be estimated [Courant, 4]. However, a careful study has shown that the fringe field of the magnets plays has an important effect both on the spin rotation and on the orbits. Then, a numerical design of the magnets by integration of the equations of motion and of the equation of spin through the magnetic field including the fringe field has been performed. The following describes results of the numerical calculations. To first order, the results agree with the analytical estimates.

The field of an infinitely long helix has been first described analytically by Blewett and Chasman [5], and then by Ptistsin [6]. Other descriptions derive the field as a superposition of undulators [Luccio, 7] or from the twisting of a cosine dipole [Caspi, 8]. For the fringe field we have simply assumed that the field in the body of the magnet will continue outside with the same helical structure and decay as a 1/cosh function in a distance equal to the radius of the magnet aperture. The actual field and its fringe will be better known when a magnet prototype is built and measured. In particular the fringe will be also affected by the method in which the super conducting magnet coils are terminated. At that time the present calculations will require some 
adjustment. For this purpose the tracking code has been written in a way to accept as input either an analytical or a mapped field.

\section{Formalism}

The helical field used in the calculation is the Blewett-Chasman field expanded to third order in the Cartesian coordinates $x$ (radial) and $y$ (vertical). The longitudinal coordinate is $z$, the axis of the helix. The components of the field are

$$
\left\{\begin{array}{l}
b_{x} \approx\left[-1-\frac{1}{4}\left(3 u^{2}+v^{2}\right)\right] \sin k z+\frac{1}{2} u v \cos k z \\
b_{y} \approx\left[1+\frac{1}{4}\left(u^{2}+3 v^{2}\right)\right] \cos k z-\frac{1}{2} u v \sin k z \\
b_{z} \approx-\sqrt{2}\left[v+\frac{1}{4}\left(u^{2} v+v^{3}\right)\right] \sin k z-\sqrt{2}\left[u+\frac{1}{4}\left(u^{3}+u v^{2}\right)\right] \cos k z
\end{array}\right.
$$

with

$$
b=\frac{B}{B_{0}} \quad\left\{\begin{array}{l}
u=k x / \sqrt{2} \\
v=k y / \sqrt{2}
\end{array} \quad k=\frac{2 \pi}{\lambda} .\right.
$$

$B_{0}$ is the maximum field on axis, $\lambda$ the field rotation wavelength.

Within the magnet aperture, the above expansion is very accurate, as compared with the complete analytical expression in terms of Bessel functions. It should be noted that the longitudinal component of the field is zero on axis but is not negligible along the deformed trajectory followed by a particle through the magnet.

The differential equation of motion in a pure magnetic field is

$$
\frac{\mathrm{d} \beta}{\mathrm{d} t}=\beta \times \Omega
$$

where $\beta$ is the relativistic velocity factor and the definitions hold

$$
\Omega=\frac{e \mathbf{B}}{m \gamma} \quad \beta^{2}=1-\frac{1}{\gamma^{2}} \quad \gamma=E / m c^{2} .
$$

The BMT differential equation for the spin precession [9] is

$$
\frac{\mathrm{d} \mathbf{s}}{\mathrm{d} t}=C_{1} \mathrm{~s} \times \Omega+C_{2}(\beta \cdot \Omega) \mathbf{s} \times \beta
$$

with the constants

$$
C_{1}=1+G \gamma \quad C_{2}=-\frac{G \gamma^{2}}{1+\gamma} \quad G=\frac{1}{2} g-1
$$

In Eq. (5) the spin is treated as an ordinary three-dimensional vector.

Using $z$ as the independent variable, and the following definitions

$$
\frac{\mathrm{d} z}{\mathrm{~d} t}=\beta_{z} c \quad \beta_{z}=\frac{\beta}{\sqrt{1+x^{\prime 2}+y^{\prime 2}}} \quad\left\{\begin{array}{l}
x^{\prime}=\beta_{x} / \beta_{z} \\
y^{\prime}=\beta_{y} / \beta_{z}
\end{array}\right.
$$

the scalar equations of motion are 
(8)

$$
\left\{\begin{array}{l}
\frac{d x^{\prime}}{d z}=x^{\prime} y^{\prime} \Omega_{z}-\left(1+x^{\prime 2}\right) \Omega_{y}+y^{\prime} \Omega_{z} \\
\frac{d y^{\prime}}{d z}=\left(1+y^{\prime 2}\right) \Omega_{x}-x^{\prime} y^{\prime} \Omega_{y}-x^{\prime} \Omega_{z} \\
\frac{d x}{d z}=x^{\prime} \\
\frac{d y}{d z}=y^{\prime}
\end{array}\right.
$$

Similarly, the scalar spin equations are

$$
\frac{\mathrm{d} s}{\mathrm{~d} z}=-\mathrm{p} \times \mathbf{s}, \quad \text { or } \quad\left\{\begin{array}{l}
\frac{\mathrm{d} s_{x}}{\mathrm{~d} z}=p_{z} s_{y}-p_{y} s_{z} \\
\frac{\mathrm{d} s_{y}}{\mathrm{~d} z}=p_{x} s_{z}-p_{z} s_{x} \\
\frac{\mathrm{d} s_{z}}{\mathrm{~d} z}=p_{y} s_{x}-p_{x} s_{y}
\end{array}\right.
$$

with

$$
\left\{\begin{array}{l}
p_{x}=C_{1} \Omega_{x} / \beta_{z}+C_{2} x^{\prime} \Gamma \\
p_{y}=C_{1} \Omega_{y} / \beta_{z}+C_{2} y^{\prime} \Gamma \\
p_{z}=C_{1} \Omega_{z} / \beta_{z}+C_{2} \Gamma
\end{array} \quad \Gamma=\beta_{z}\left(x^{\prime} \Omega_{x}+y^{\prime} \Omega_{y}+\Omega_{z}\right) \quad s_{z}^{2}+s_{y}^{2}+s_{z}^{2}=1\right.
$$

To calculate the direction of the axis around which the spin precesses in its traversal of a spin rotator, we use 3 more equations, formally identical to (9), i.e.

$$
\frac{d s^{A}}{d z}=-p \times s^{A}
$$

The idea -admittedly not too elegant- is the following: if a spin vector $\mathbf{s}$ precesses from $\mathrm{s}_{0}$ to $\mathbf{s}_{\mathrm{f}}$, the axis of precession must belong to the plane $\pi$ bisecting the $\left(\mathbf{s}_{0} \mathbf{s}_{\mathrm{f}}\right)$ angle, as shown in Fig. 1 . The axis is then perpendicular to the vector $\mathbf{d s}=\mathbf{s}_{\mathbf{f}}-\mathbf{s}_{0}$. If we repeat the same argument for a second spin orientation $\mathbf{s}^{\mathrm{A}}$, the axis must be also perpendicular to $d s^{A}=\mathbf{s}_{\mathbf{f}}{ }^{\mathrm{A}}-\mathbf{s}_{0} \mathrm{~A}$. The axis of precession $\mathbf{s}$ is therefore perpendicular both to ds and to ds ${ }^{\mathrm{A}}$.

$$
\sigma=\delta s \times \delta s^{A}
$$

and the angles, as defined in figure 2 are

$$
\left\{\begin{array}{l}
\tan \phi=\frac{\sigma_{x}}{\sigma_{z}} \\
\tan \theta=\frac{\sigma_{y}}{\sqrt{\sigma_{x}^{2}+\sigma_{z}^{2}}}
\end{array}\right.
$$

In total, we integrate 10 differential equations. 4 equations for the trajectory, Eqs. (8) and 6 equations for the spin, Eqs. (9), with two starting orientations of the spin, that will enable us to calculate the axis of precession.

The integration is performed by a code, SNIG [10], employing a Hamming PredictorCorrector third order solver with automatic adjustment of the integration step. 


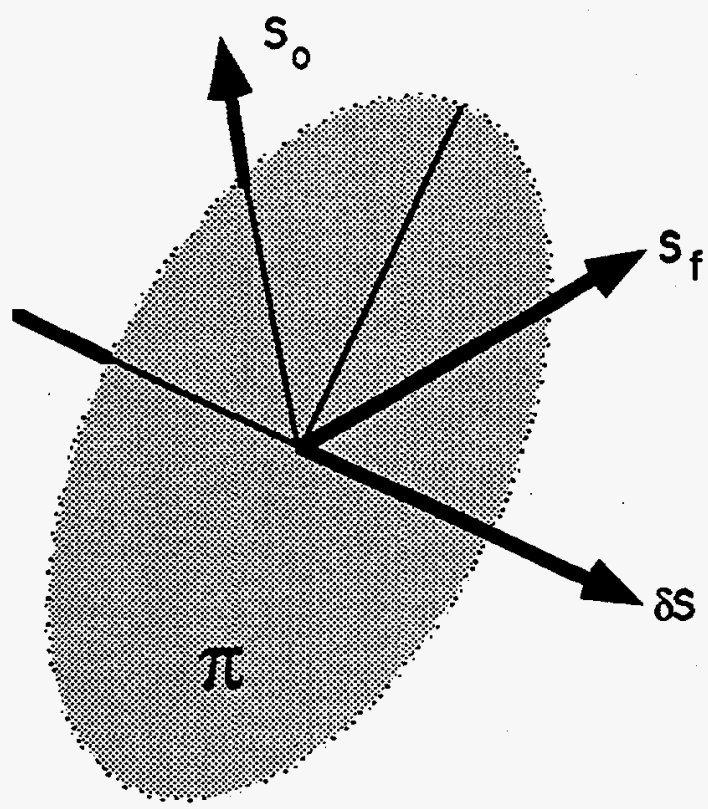

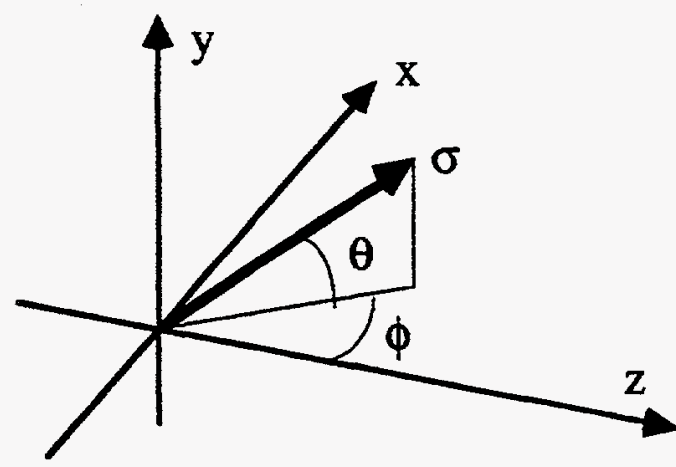

Fig. 2. Angles of the axis of precession. $\phi$, horizontal, $\theta$, vertical.

Fig. 1. To find the axis of precession in a snake

\section{Results. Spin Rotator.}

Spin rotators for RHIC must rotate the spin by $90^{\circ}$, from the vertical direction $y$ to the horizontal $x-z$ plane. For experiments, collisions between counter streaming proton beams in the machine insertions will be done at every energy, from injection, $\gamma=27$, to the maximum energy, $\gamma=250$. In the collisions we want the spin in the longitudinal, or $z$, direction or in the radial, $x$ direction. An arrangement that satisfies this requirements consists of four super conducting helical magnets, alternately right-handed and left-handed, with a maximum field close to 4 Tesla. Geometric spin rotator parameters are listed in Table 1. Fig. 3 shows the three components of the magnetic field and the orbit, at injection energy. The magnetic field shown is evaluated along the orbit, as seen by the proton. A result of spin tracking with SNIG through a spin rotator is also shown in Fig. 3.

Table 1. Parameters of the Spin Rotators magnets.

\begin{tabular}{|c|c|c|c|}
\hline \multicolumn{3}{|c|}{$\begin{array}{l}\text { Number of helical magnets } \\
\text { Total length }[\mathrm{m}] \\
\text { Magnet bore }[\mathrm{mm}]\end{array}$} & $\begin{array}{c}4 \\
10.56 \\
100 \\
\end{array}$ \\
\hline \multicolumn{4}{|c|}{ Helical magnets } \\
\hline Length $[\mathrm{m}]$ & Field & Field rotation ${ }^{\mathrm{a}}[\mathrm{deg}]$ & Field orientation ${ }^{b}[\mathrm{deg}]$ \\
\hline 2.40 & B1 & +345 & 97.5 \\
\hline 2.40 & B2 & -345 & 82.5 \\
\hline 2.40 & $\mathrm{~B} 2$ & +345 & 97.5 \\
\hline 2.40 & $\mathrm{B1}$ & -345 & 82.5 \\
\hline
\end{tabular}

Notes:

a "+": right-handed, "-" : left-handed helix.

$b$ Field angle respect to the vertical at magnet's entry. 


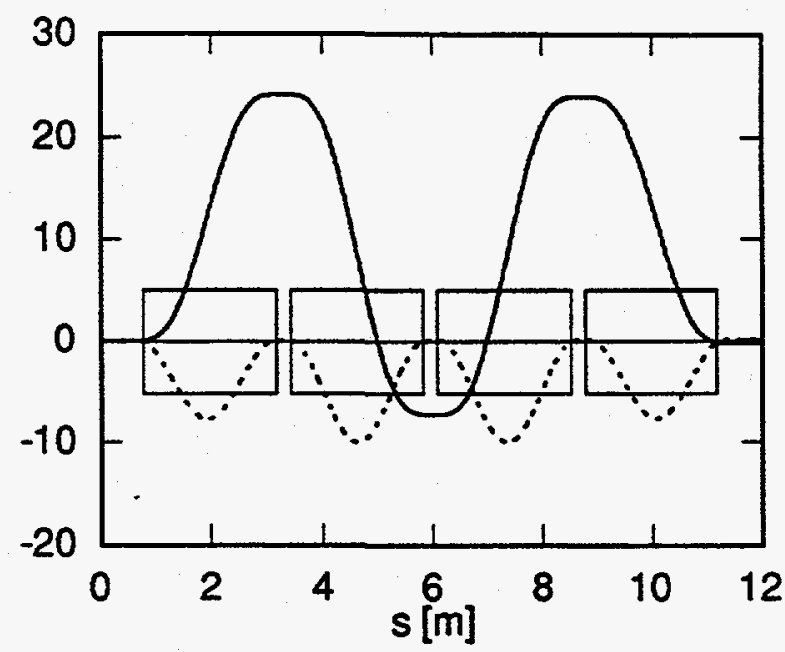

Fig. 3a. 4-Helix spin rotator. Orbit [mm] at $\gamma=27$.

Solid line, horizontal trajectory. Dotted, vertical.

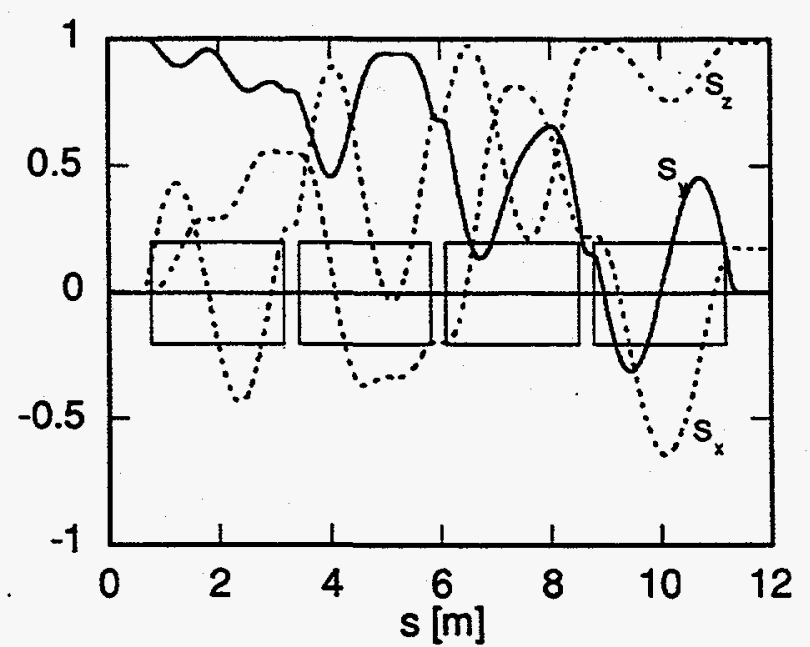

Fig. 3b. 4-Helix spin rotator.

Spin tracking. In the rotator the polarization is brought from vertical to horizontal.

Case for longitudinal polarization.

The angle of rotation shown in Table 1 is $345^{\circ}$. Now, according to the original theory [3], in each magnetic module the transverse field rotates a full $360^{\circ}$ as one progresses to the other side. However in this case, if the fringe field is taken into account, because of the anti symmetric structure of a spin rotator the field integrals cannot be made equal to zero. Fig. $3 \mathrm{c}$ clearly shows that the integrals of the fringe field in general do not cancel. The result is that a particle entering in the magnet on axis will not in general emerge on axis. In our design the problem was solved by making the field rotation less than $360^{\circ}$, so the field integrals can be exactly compensated at least for one beam energy.

In RHIC the direction of the beam line where a spin rotator will be placed is at an horizontal angle $\phi=3.674 \mathrm{mrad}$ with the direction of the adjacent insertion [11]. The spin, after the rotator, will perform a further precession in the horizontal plane. Then, to achieve longitudinal polarization at the insertion, the spin should emerge from the rotator in the horizontal plane and at an angle $G \gamma \phi$ or $G \gamma \phi \pm \pi$ with the rotator axis, in the positive direction of the axis $z$ (forward) or negative (backward), respectively. The corresponding angles for radial polarization are $G \gamma \phi \pm$ $\pi / 2$ in the negative direction of the axis $x$ (inward) or positive (outward), respectively. 


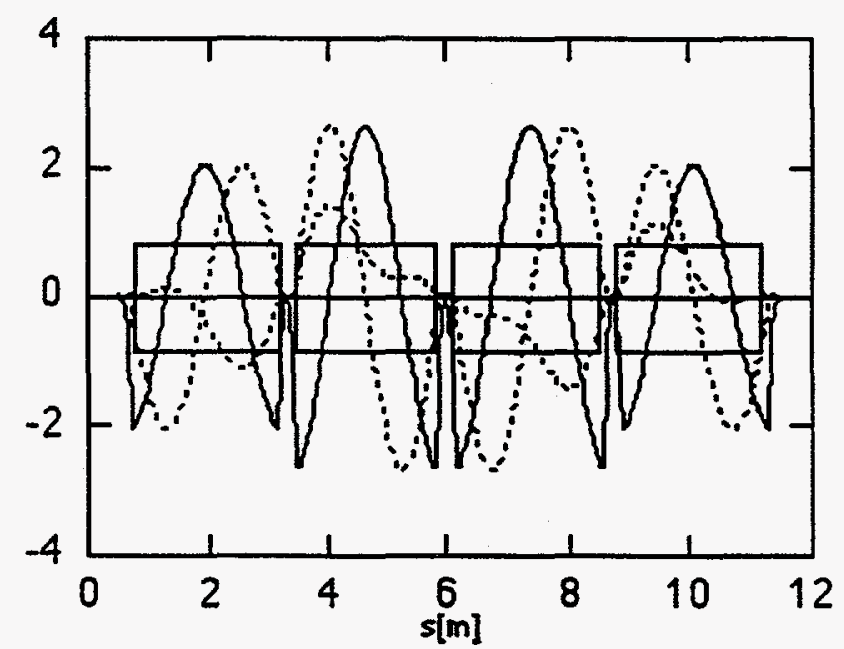

Fig. 3c. 4-Helix spin rotator. Field [tesla] at $\gamma=27$.

Solid line, vertical field. Dotted, horizontal and longitudinal.

The longitudinal field is on scale $1: 10$.

The angles are given by

$$
\tan ^{-1}\left(\frac{S_{x}}{S_{z}}\right)=\left\{\begin{array}{c}
G \gamma \phi \\
G \gamma \phi \pm \pi . \\
G \gamma \phi \pm \pi / 2
\end{array} .\right.
$$

Eq. (14) shows that the needed spin rotation is dependent on beam energy. Fig. 4 shows the loci of $S_{y}=0$ on a $B_{1}-B_{2}$ plane. Each point on the curves corresponds to a value of the angle of Eq. (14). From this diagram we can find the values of the field needed to provide a

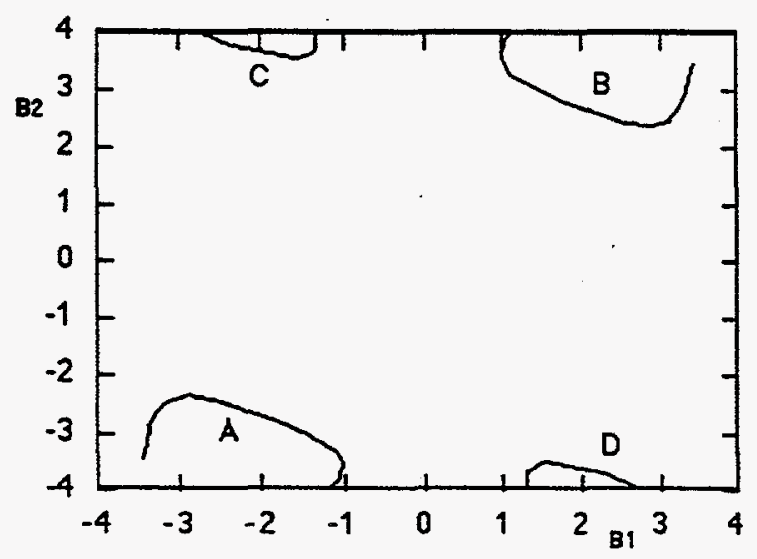

Fig. 4. 4-Helix spin rotator. Loci of $S_{y}=0$.

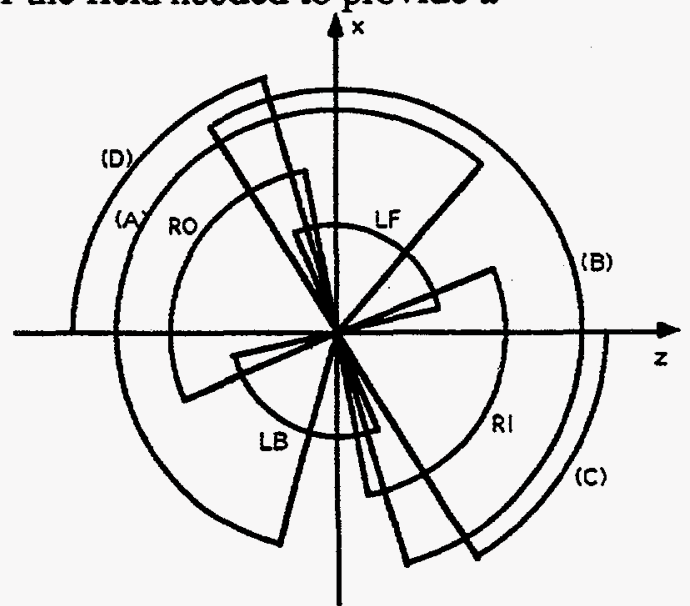

Fig. 5. 4-Helix spin'rotator. Angles required for longitudinal and radial polarization:

LF, long. forward, LB, long. backwards, RO, radial outwards, RI, radial inwards.

Rotator capabilities:

A, B, C, D refer to the "islands" of Fig. 5.

Angles of $B$ are good for $L F$ and angles of $A$ for $R O$. 
longitudinal or radial polarization at different energies The spin angles obtainable with the rotator are also shown in Fig. 6. In the figure the required angles are also shown. Fig. 6 shows that the spin rotator can provide a longitudinal forward polarization and a radial outward polarization for all proton energies in the range, with field not greater than 4 Tesla, but not all angles for longitudinal backwards and radial inwards.

The values of the field and of other parameters of the rotator are given in Tables 2 and 3, for longitudinal forward and radial outward polarization, respectively. The field is also shown in Figs. 7 and 8.

A comparison of Tables 2 and 3 shows that the 4-helix spin rotator is particularly effective ito achieve a longitudinal polarization, but less for radial polarization, as values of the maximum orbit excursion show. However, for experiments with colliding polarized beams, the longitudinal polarization is more important than the radial, and the for-vs. backwards or out- vs. inwards question has no practical relevance [12].

Table 2. Spin Rotator for longitudinal polarization "forwards".

\begin{tabular}{|c|c|c|c|c|c|c|c|}
\hline Beam energy, $g$ & 27 & 50 & 75 & 100 & 150 & 200 & 250 \\
\hline$G x \phi$ & 10.19 & 18.70 & 28.31 & 37.75 & 56.63 & 75.49 & 113.23 \\
\hline & \multicolumn{7}{|c|}{ Field $[\mathrm{T}]$} \\
\hline B1 & 2.047 & 2.281 & 2.528 & 2.752 & 3.082 & 3.257 & 3.354 \\
\hline B2 & 2.654 & 2.543 & 2.439 & 2.373 & 2.421 & 2.679 & 3.005 \\
\hline & \multicolumn{7}{|c|}{ Max. orbit excursion [mm] } \\
\hline horiz. & 24.096 & 14.495 & 10.708 & 8.741 & 6.527 & 5.172 & 4.263 \\
\hline vertical & 10.015 & 5.191 & 3.459 & 2.825 & 2.110 & 1.673 & 1.379 \\
\hline & \multicolumn{7}{|c|}{ Field integrals $[\mathrm{T}-\mathrm{m}]$} \\
\hline $\int\left|B_{x}\right| d s$ & 15.005 & 15.389 & 15.847 & 16.346 & 17.551 & 18.931 & 20.284 \\
\hline $\int\left|B_{y}\right| d s$ & 15.010 & 15.398 & 15.853 & 16.356 & 17.562 & 1.943 & 20.297 \\
\hline $\int\left|B_{2}\right| d s$ & 0.552 & 0.312 & 0.240 & 0.208 & 0.170 & 0.144 & 0.125 \\
\hline & \multicolumn{7}{|c|}{ Orbit lengthening [mm] } \\
\hline & 1.252 & 0.378 & 0.178 & 0.107 & 0.055 & 0.036 & 0.026 \\
\hline
\end{tabular}

Table 3. Spin Rotator for radial polarization "outwards".

\begin{tabular}{|c|c|c|c|c|c|c|c|}
\hline Beam energy, $g$ & 27 & 50 & 75 & 100 & 150 & 200 & 250 \\
\hline$G x+\pi / 2$ & 100.19 & 108.70 & 118.31 & 127.75 & 146.63 & 165.49 & -156.77 \\
\hline & \multicolumn{7}{|c|}{ Field [T] } \\
\hline B1 & -3.293 & -3.234 & -3.145 & -3.023 & -2.652 & -2.162 & -1.222 \\
\hline B2 & -2.732 & -2.603 & -2.471 & -2.384 & -2.398 & -2.602 & -3.171 \\
\hline & \multicolumn{7}{|c|}{ Max. orbit excursion [mm] } \\
\hline horiz. & 38.754 & 20.548 & 13.321 & 9.603 & 5.617 & 3.433 & 2.477 \\
\hline vertical & 12.423 & 6.599 & 4.296 & 3.102 & 1.817 & 1.330 & 1.295 \\
\hline
\end{tabular}




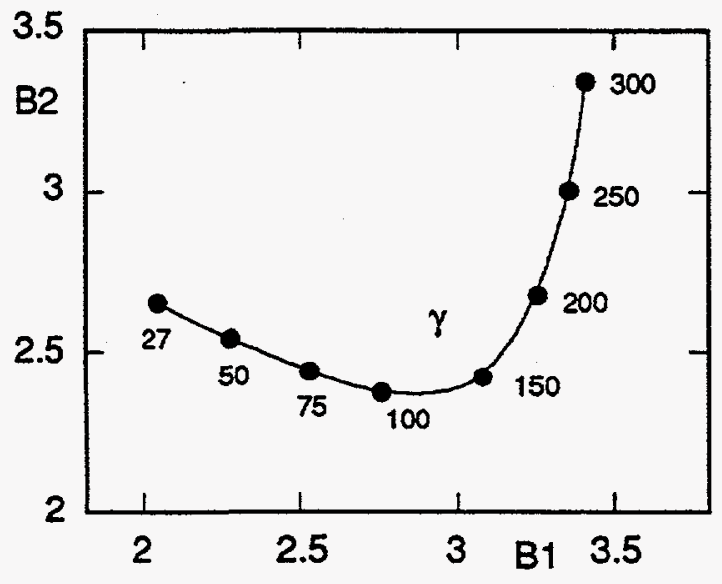

Fig. 6. 4-Helix rotator. Field [tesia] in the two pairs of magnets to achieve longitudinal polarization in the insertion of RHIC, for various beam energies.

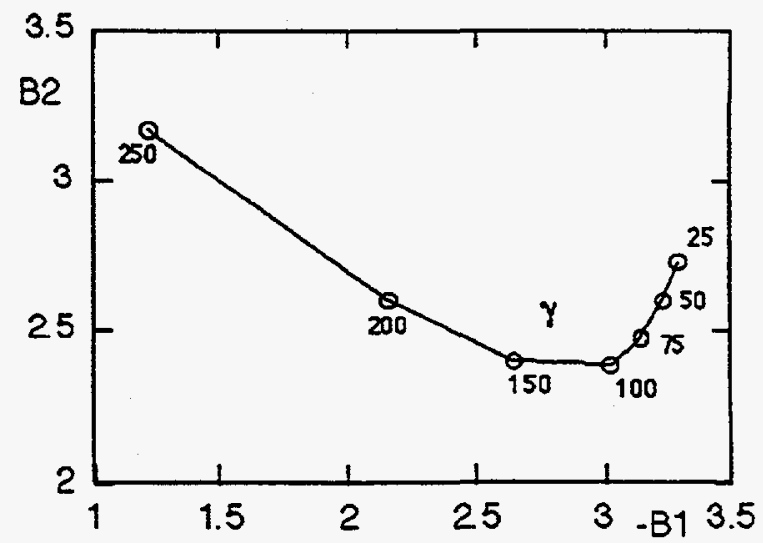

Fig. 7. 4-Helix rotator. Field [tesla] in the two pairs of magnets to achieve radial polarization in the insertion of RHIC, for various beam energies.

\section{Results. Siberian Snake.}

Siberian snakes for RHIC must rotate the spin by $180^{\circ}$, from the vertical up to the vertical down. The axis of precession should make an angle $\phi= \pm 45^{\circ}$ with the beam (Eq. 13). This angle should be slightly adjustable to compensate for misalignments and for possible spin rotation created by the solenoidal fields in the detectors. An arrangement that satisfies this requirement consists of four super conducting helical magnets, right-handed, with a maximum field of 4 Tesla.

Geometric Siberian snake parameters are listed in Table 4. Fig. 8 shows the three components of the magnetic field, the orbit, and the spin precess-ion at injection energy. The symmetry of the Siberian snake is such that fringe field would cancel in any case. However, in order to simplify the construction of the snakes/rotators, a solution has been worked out with all magnetic modules identical in both devices (apart from their right- or left-handedness).

All helical magnets are powered by separate power supplies. This allow for an adjustment of the spin tune to $1 / 2$ and also for small changes of the direction of the precession axis to compensate for the effect of the detector solenoids. This is shown in Fig. 9.

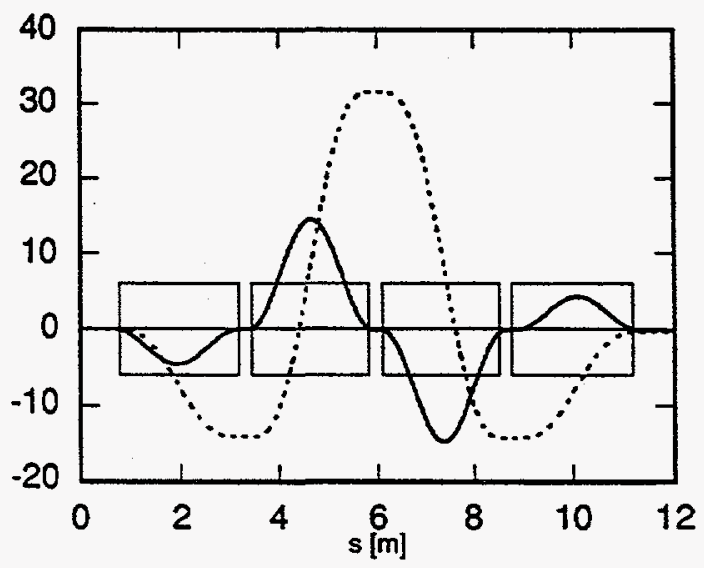

Fig. 8a. 4-Helix snake. Orbit $[\mathrm{mm}]$ at $\gamma=27$.

Solid line, horizontal trajectory. Dotted, vertical. Case for precession axis at $45^{\circ}$. 


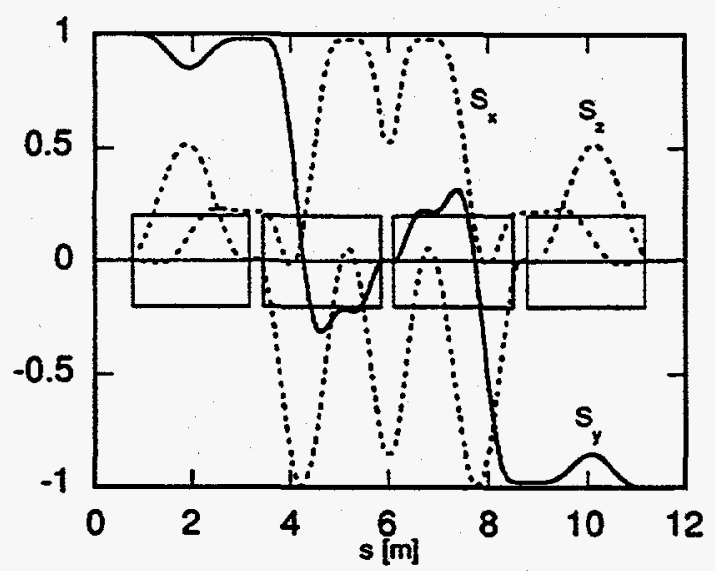

Fig. 8b. 4-Helix Siberian Snake. Spin tracking at $y=27$.

In the sanke the polarization is flipped vertically. Case for precession axis at $45^{\circ}$.

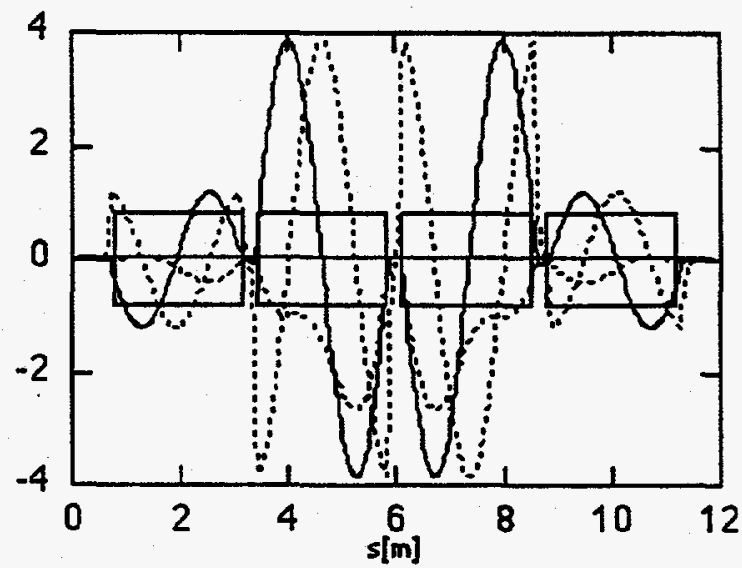

Fig. 8c. 4-Helix Snake. Field [tesla] at $\gamma=27$, precession axis at $45^{\circ}$.

Solid line, vertical field. Dotted, horizontal and longitudinal.

The longitudinal field is on scale 1:10.

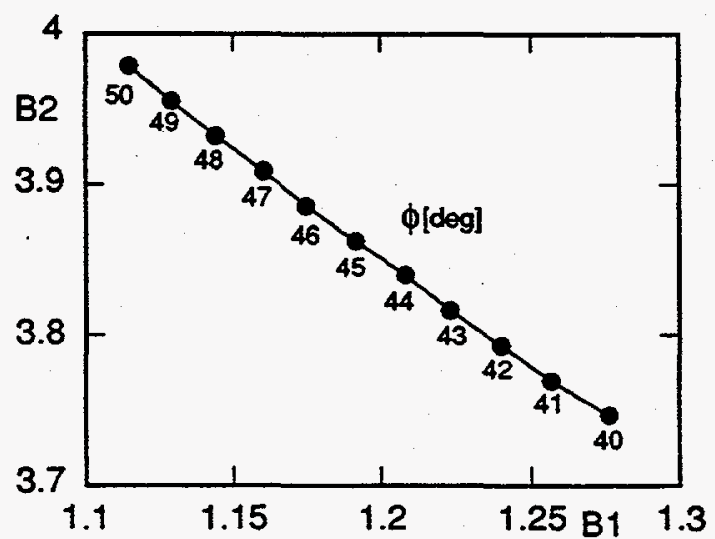

Fig. 9. 4-Helix Siberian Snake.

Change of the horizontal angle of the spin precession axis (around $45^{\circ}$ ) as a function of the field. 
Table 4. Parameters of the Siberian Snake magnets.

\begin{tabular}{l}
$\begin{array}{l}\text { Number of helical magnets } \\
\text { Total length [m] } \\
\text { Magnet bore [mm] }\end{array}$ \\
\hline
\end{tabular}

Notes: ${ }^{2}$ "+ ${ }^{n}:$ right-handed, ${ }^{n}$ " : left-handed helix.

$b$ Field angle respect to the vertical at magnet's entry.

c For axis of spin precession at $45^{\circ}$.

d@ injection, $\mathrm{g}=27$.

\section{Optical Transfer Matrix}

We have calculated the optical transfer matrix up to the third order with SNIG. This was done by tracking a certain number of particles -typically 35- with coordinates randomly extracted inside a phase space ellipse with the appropriate Twiss parameters for RHIC. The output of the tracking is then fitted with a third order polynomial in $x, y, x^{\prime}$ and $y^{\prime}$ and the coefficients of a first, second, and third order matrix are found. The first order matrix elements are shown in Table 5 (for the snake of Table 4). They are in good agreement with the corresponding approximate analytical calculations $[4,13]$. We use more particle than are strictly needed, and the fitting is repeated several times with all possible combination of the particles. So, the matrix elements shown are in reality averages with statistical variance.

The final spin values are also calculated and averaged, to determine how the spin rotation is affected by the finite size of the beam. They are shown in Table 6. It is apparent that the spin reversal is not affected by the size of the beam.

Table 5. Transfer matrix for a 4-Helix snake.

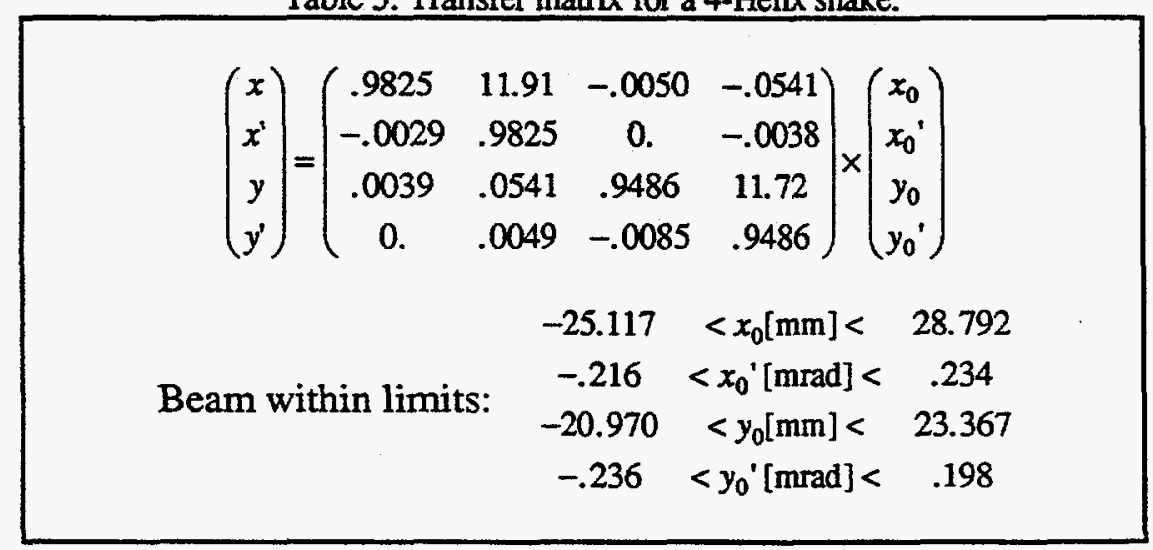


Table 6. 4-Helix Siberian Snake. Spin tracking

\begin{tabular}{|c|c|c|c|}
\hline & Sx & Sy & Sx \\
\hline start spin & 0 & 1 & 0 \\
\hline final spin & $-8.2710^{-5}$ & -1 & $4.0710^{-4}$ \\
\hline with variance & $5.1710^{-13}$ & $2.1510^{-9}$ & $3.6110^{-12}$ \\
\hline
\end{tabular}

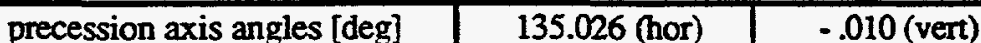

\begin{tabular}{|l|l|l|}
\hline with variance & $1.0010^{-6}$ & $5.8710^{-11}$ \\
\hline
\end{tabular}

\section{Conclusions}

Spin rotators and Siberian snakes for RHIC can be built using 4 helical magnets. Although super conducting helical magnets of this size have never been build, they can by obtained, by twisting, from the super conducting cosine dipoles being built for RHIC. So, we are confident that they will not present hard problems. We found that the fringe fields are important, and they should be properly taken into account. In the calculations we have used a model for those fringes that is plausible, but only magnetic measurements on the prototypes being built will allow a final optimization. To play safe, we have repeated calculations in two extreme cases: i.e. hard edge magnets with no fringe and a fringe that extends outside the magnets to a much larger distance. The results appear conveniently bracketed by extreme cases calculations, giving us added confidence.

As we mentioned, prototypes are being built and will be measured. The code SNIG has been written with the option to integrate the equations of motion and the equations of spin using either an analytical expression for the magnetic field or by three dimensional interpolation in a magnetic field map. At the appropriate time, new results will be issued.

\section{Acknowledgments}

Basic parameters for the Snakes and rotators are the result of many discussions mostly with E.D.Courant, S.Peggs, V.Ptitsin, T.Roser and E.Willen, not necessarily in that order.

\section{References}

[1] Conceptual Design for the Acceleration of Polarized Protons in RHIC, RSC Collaboration (T.Roser, Spokesperson). May 24, 1993, Rev. February 15, 1995

[2] Polarized Collider Workshop University Park, PA 1990(J.Collins, S.F.Heppelman, R.W.Robinett, Eds.) A.I.P. conference Proceedings No. 223 also: A.Luccio and M. Conte, Wiggler s as Spin Rotators for RHIC, Proc. IEEE Part. Acc. Conf. Washington, DC, May 17-20, 1993, Vol.I, p.438

[3] V.I.Ptitsin and Yu.M. Shatunov, Helical Spin Rotators and Snakes, Proc. Third Workshop on Siberian Snakes and Spin Rotators (A.Luccio and Th.Roser Eds.) Upton, NY, Sept. 12-13,1994, BNL-52453, p.15

[4] E.D.Courant, RHIC/AP/47

[5] J.P.Blewett and R. Chasman, J.Ap.Phys, 48 (1977) 2692

[6] V.I.Ptitsin RHIC/AP

[7] A.Luccio, in "Halbach Festschrift" LBL, PUB-754, Vol.1, p.65, Feb. 1995

[8] S.Caspi, LBL-35928, July 1994

[9] J.D.Jackson, Classical Electromagnetism, Wiley, NY, 2.nd ed 1962, p.558

[10] A.Luccio, Program SNIG, unpublished, and SNIG Formalism

Proc. Third Workshop on Siberian Snakes, loc. cit., p. 193

[11] RHIC design Manual, Rev. May 1994. "Lattice and Beam Dynamics" p.7

[12] T.Roser, Private Communication 